\title{
Digitale Beschaffung und Anforderungen im Unterschwellenbereich
}

Die elektronische Vergabe für Aufträge oberhalb der europäischen Schwellenwerte im öffentlichen Sektor ist bereits zum Standard geworden. Die E-Vergabe im Unterschwellenbereich steht nun an und fordert von den Trägern öffentlicher Krankenhäuser die Einführung digitaler Vergabeprozesse.

Die Umsetzung der Digitalisierung hat begonnen und ist von öffentlichen Auftraggebern zwingend zu beachten. Während Beschaffungsinstitutionen und Wirtschaft noch einheitliche Regelungen zur Umsetzung einer gewünschten Digitalisierung erarbeiten, sind öffentliche Auftraggeber seit Oktober 2018 gesetzlich verpflichtet, Aufträge oberhalb des europäischen Schwellenwertes digital auszuschreiben. Mit der Reform des Vergaberechts wurden unmittelbar Anforderungen an den Einsatz elektronischer Mittel im Vergabeverfahren nach Paragraf 11 Vergabeverordnung $(\mathrm{VgV})$ geregelt. Während die Rechtslage im Bereich der EU-weiten Ausschreibungen bezüglich der Umsetzung elektronischer Vergaben (sogenannter E-Vergaben) damit bereits manifestiert ist, steht die flächenweite Umsetzung der E-Vergabe auch für den Unterschwellenbereich an.

\section{Zeitliche Komponenten der} Digitalisierung

Nach den aktuellen Regeln der Unterschwellenvergabeverordnung (UVgO) haben Auftraggeber noch eine Wahlfreiheit dahin gehend, ob die Unternehmen ihre Teilnahmeanträge und Angebote in Textform nach Paragraf 126b des Bürgerliches Gesetzbuchs (BGB) mithilfe elektronischer Mittel einzureichen haben (Paragraf 38 Absatz 1 UVgO). Spätestens seit dem 1. Januar 2019 müssen Auftraggeber nun grundsätzlich die Einreichung von Teilnahmeanträgen und Angeboten mithilfe elektronischer Mittel akzeptieren, selbst wenn sie eine andere Übermittlungsform vorgegeben haben. Dies beinhaltet, dass Auftraggeber spätestens seit dem 1. Januar

\section{Ab dem Jahr 2020 gilt die E-Vergabe für alle Ausschrei- bungen - auch im Unterschwellen- bereich.}

eine elektronische Vergabeplattform vorhalten müssen. Der nächste Meilenstein erfolgt dann ab dem 1. Januar 2020. Ab diesem Zeitpunkt gilt dann, ebenso wie im Bereich der EU-weiten Ausschreibungen, die Pflicht, auch Unterschwellenvergaben volldigitalisiert durchzuführen.

\section{Bedeutung für öffentliche Auftraggeber}

Tatsächlich sagen Schätzungen aus, dass nur zehn Prozent aller öffentlichen Auftragsvergaben den EU-Schwellenwert erreichen oder überschreiten. Daraus folgt der Rückschluss, dass der Großteil aller Vergaben zu den Unterschwellenvergaben gehören. Der Bedeutung der Digitalisierung für das öffentliche Auftragswesen ist damit ein höheres Gewicht zuzumessen, da mit dieser Regelung breitflächig für die überwiegende Anzahl auszuführender Vergabeverfahren neue technische Voraussetzungen gefordert werden.

\section{Sachstand Umsetzung UVgO}

Die zeitliche Festsetzung hat ihren Niederschlag in Paragraf 38 Absatz 3 UVgO gefunden. Die Umsetzung der Unterschwellenvergabeordnung in den einzelnen Bundesländern kommt langsam aber stetig voran. Die Regelung gilt für die Vergabe von öffentlichen Liefer- und Dienstleistungen unterhalb der EU-Schwellenwerte und ersetzt die bisher geltende Vergabe- und Vertragsordnung VOL/A, Abschnitt 1. Sie orientiert sich an der für öffentliche Aufträge ab Erreichen oder Überschreiten der Schwellenwerte geltenden Vergabeverordnung und verweist zudem an einigen Stellen auf die Bestimmungen des Teils 4 des Gesetzes gegen Wettbewerbsbeschränkungen (GWB).

Die UVgO als Verfahrensordnung ist weder Gesetz noch Rechtsverordnung. Die Verpflichtung zur Anwendung erfolgt erst durch einen entsprechenden Anwendungsbefehl. Das heißt, erst die Bekanntmachung im Bundesanzeiger entfaltet diese Rechtsbindung. Unter dieser Maßgabe ist auch erklärlich, dass bis heute die Umsetzung der Anwendung nicht vollständig und insbesondere nicht zeitgleich in allen Bundesländern erfolgt ist. Exemplarisch ist festzustellen, dass Berlin die UVgO erst 2019 einführen wird. Das Wirtschaftsministerium Rheinland-Pfalz spricht von einer geplanten Neufassung der Verwaltungsvorschrift, nennt aber kein Zeitziel. Ein erster Entwurf sollte Ende 2018 vorliegen. In Niedersachsen war die Anpassung auf die Zeit nach der Landtagswahl verschoben worden, die allerdings bereits im Oktober 2017 vollzogen war. Derzeit gibt es bezogen auf die Einführung der UVgO noch keine Informationen der Bundesländer Hessen und Sachsen-Anhalt.

Ohne einen entsprechenden Anwenderbefehl ist eine Umsetzung der Vorgaben 


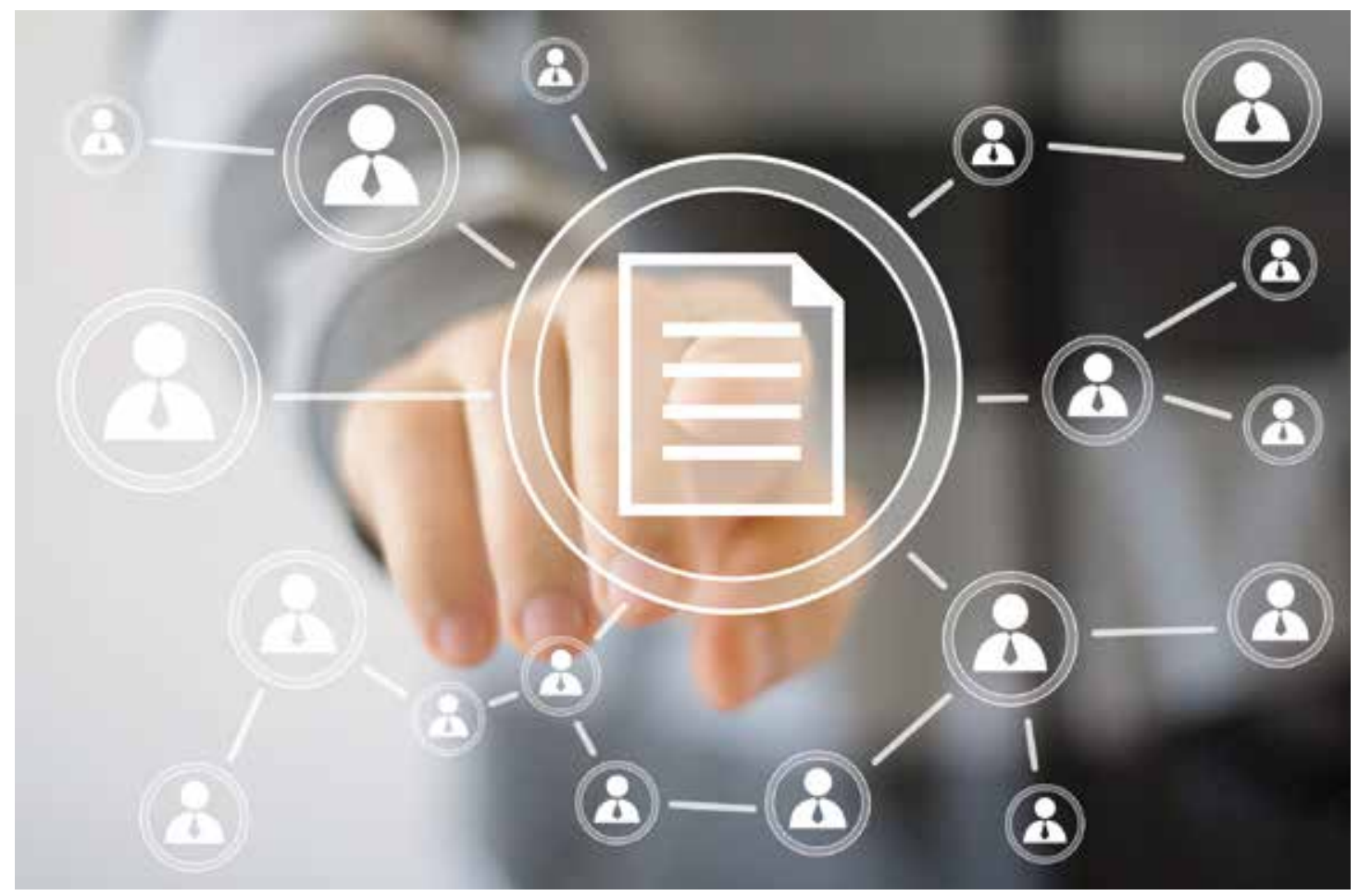

Symbolfoto: AdobeStock/maxsim

Relevante gesetzliche

Vorgaben:

- Vorgaben für digitale Auftragsvergabe:

- §11 der Vergabeverordnung $(\mathrm{VgV})$

Zur Form der Angebotsabgabe und Kommunikaiton:

- §38 Absatz 1 Unterschwellenvergabeordnung (UVgO)

- §126 b Bürgerliches Gesetzbuch (BGB)

Entscheidung der Vegabekammern

- VK-Bund, Beschluss vom 19.07.2018 - VK 2-58/18

- VK Lüneburg, Beschluss vom 08.05.2018 - VgK-10/2018

auf Länderebene nicht gegeben. Unter der Maßgabe der vorgenannten uneinheitlichen Umsetzung bestehen daher derzeit auf Bundesebene unterschiedliche rechtliche Voraussetzungen für den Unterschwellenbereich, welche sich v.a. auf die
Umsetzung der E-Vergabe auswirken. Öffentliche Auftraggeber aus Bundesländern, in denen eine Anwendbarkeit der UVgO noch nicht umgesetzt ist, werden von den Verpflichtungen zur Digitalisierung entsprechend Paragraf 38 UVgO nicht erfasst. Hier gilt weiter die alte Rechtslage und damit die Möglichkeit, im Unterschwellenbereich in Papierform auszuschreiben.

Auch wenn deutlich ist, dass sich eine vollständige Umsetzung der Anwendbarkeit der E-Vergabe-Vorgaben aus der UVgO auf Länderebene nicht aufhalten lässt, birgt die gegebene Situation eine Rechtsunsicherheit der Anwender.

\section{Folgen der zeitversetzten Umsetzung}

Auch wenn einzelne Bundesländer erst zeitverzögert die Anwendungsbefehle zur UVgO verabschieden, so gelten ab dem Zeitpunkt der Verabschiedung die Regelungen unmittelbar. Wähnen sich öffentliche Auftraggeber derzeit in einzelnen Ländern bezogen auf die Schaffung der Voraussetzung zur E-Vergabe noch in einem sicheren Rechtsgefüge, so werden sie mit der entsprechenden Verabschiedung der gesetzlichen Voraussetzungen ohne weitere Vorankündigung in die digitale Welt gestoßen.
Öffentliche Auftraggeber, die hiervon betroffen sind, müssen dann ohne Vorlauf die Einreichung von Teilnahmeanträgen und Angeboten in Textform mithilfe elektronischer Mittel ermöglichen (Paragraf 7 UVgO), auch wenn eine andere klassische Übermittlungsform vorgegeben war. Grundsätzlich ist dazu die Vorhaltung einer entsprechenden Vergabeplattform notwendig. Treffen diese Voraussetzung die betroffenen öffentlichen Auftraggeber unvorbereitet, so kann es zu erheblich zeitverzögerten Beschaffungsmaßnahmen kommen, welche aus der Implementierung entsprechender Systeme nebst Schulung zu begründen sind.

Ausnahmen von der elektronischen Übermittlung regeln die Paragraf 38 Absätze 4 und $5 \mathrm{UVgO}$. Eine Freistellung dieser Voraussetzungen ist dann gegeben, wenn der Auftragswert ohne Umsatzsteuer

25000 Euro nicht überschreitet oder wenn eine beschränkte Ausschreibung ohne Teilnahmewettbewerb oder eine Verhandlungsvergabe ohne Teilnahmewettbewerb durchgeführt wird. Bei diesen einstufigen Verfahren wird die zwingende Notwendigkeit einer elektronischen Vergabe nicht gesehen. 


\author{
EU-Schwellenwerte seit \\ 1. Januar 2018* \\ Angaben in Euro
}

\section{Klassische Auftragsvergabe}

Liefer- und Dienstleistungen

221000 Euro

(bisher: 209000 Euro)

Bauleistungen

5548000 Euro

(bisher: 5225000$)$

\section{Konzessionsvergabe 5548000 Euro}

\section{Auftraggeber im Sektoren bzw. Verteidigungs- und Sicherheitsbereich \\ Liefer- und Dienstleistungen \\ 443000 Euro}

Bauleistungen

5548000 Euro

Soziale und andere besondere Dienstleistungen § 130 GWB 750000 Euro

*Gültig seit 1. Januar 2018. Die Schwellenwerte werden in der Regel alle zwei Jahre überprüft.

Unabhängig vom Durchsetzungsgrad der UVgO kann daher öffentlichen Auftraggebern nur angeraten werden, bereits frühzeitig entsprechende Systeme zu implementieren und ausführende Mitarbeiter auf den Systemen zu schulen. Digitalisierung umfasst dabei nicht allein den Anschluss an eine bestehende Vergabeplattform. Es müssen auch webbasierte Adressen geschaffen werden von denen Bieter ohne Registrierung
Auftragsbekanntmachungen und Vergabeunterlagen abfordern können. In Zeiten der Datenschutzgrundverordnung (DSGVO) bedarf auch dies eines zeitlichen Vorlaufs. Diese Feststellungen erlangen umso mehr Bedeutung, wenn man nochmals auf die Tatsache reflektiert, dass die Umsetzung der E-Vergabe im Unterschwellenbereich ca. 90 Prozent aller Beschaffungsvorgänge betrifft.

Die Stolpersteine im Rahmen der E-Vergabe sind bereits heute Gegenstand von Vergabeverfahren. Hierzu sei auf die Entscheidung der Vergabekammer des Bundes beim Bundeskartellamt (VK-Bund) Beschluss vom 19. Juli 2018 hinzuweisen. Themenfeld dieser Entscheidung war in Bezug einer E-Vergabe, dass dem Bieter die Vergabeunterlagen „vollständig und direkt“ zum Abruf zur Verfügung gestellt werden müssen. Die Entscheidungen zu den Voraussetzungen einer rechtskonformen Umsetzung der digitalen Anforderungen sind bereits heute vielfältig.

\section{Rolle von Beschaffungsinstitutionen} Der Anschluss öffentlicher Auftraggeber an Beschaffungsinstitutionen stellt in diesem Zusammenhang sicherlich eine grundsätzliche Erleichterung dar. Beschaffungsinstitutionen bündeln in der Regel den Bedarf ihrer Mitglieder und müssen diesen dann, sofern dies auch von den Mitgliedern gewünscht ist, ausschreiben. Sie können dazu selbstverständlich aus den verschiedenen Verfahrensarten wählen. Gerade die Bündelung des Bedarfs der angeschlossenen Mitglieder von Beschaffungsinstitutionen verdeutlicht aber, dass deren Handeln sich in der Regel auf ein Handeln im Verbund bezieht. Darauf folgt, dass das Volumen nach positiver Bündelung in der Regel die Schwellenwerte der Beschaffungen übersteigen und Ausschreibungen sich an den Regelungen der Vergabeverordnung orientieren. Das Handeln von Beschaffungsinstitutionen umfasst daher in erster Linie Ausschreibungen im Oberschwellenbereich und nicht die verbleibenden 90 Prozent an Ausschreibungen im Unterschwellenbereich.

\section{Alternative Möglichkeiten}

In der Regel steht damit der öffentliche Auftraggeber im Bereich der vielfältigen Unterschwellenvergaben allein. Es verbleibt dann, sofern die Implementierung einer eigenen Vergabeplattform durch den öffentlichen Auftraggeber nicht gewünscht ist, allein die Möglichkeit sich auf Fremddienstleister zu berufen und die Durchführung von Vergabeverfahren in die Hände entsprechender Anbieter zu geben. Die Inanspruchnahme von Fremddienstleistern wiederum erfolgt auch entgeltlich, sodass auch diesbezüglich ebenfalls die Frage nach der Ausschreibungspflicht gegeben ist.

\section{Fazit}

Unter der Maßgabe der fortschreitenden Digitalisierung und der verpflichtenden Umsetzung der E-Vergabe im Unter- und Oberschwellenbereich ab dem 1. Januar 2020 ist daher öffentlichen Auftraggebern dringend anzuraten, auf die neuzeitlichen Anforderungen zu reagieren. Der Anteil der überwiegenden Beschaffungsvorgänge, welche sich im Unterschwellenbereich darstellen, kann nur ohne zeitliche Brüche umgesetzt werden, wenn frühzeitig die Weichen hierzu gestellt werden. Die Implementierung einer eigenen Vergabeplattform nebst Schulungen der ausführenden Mitarbeiter ist dazu ratsam. Im Gegenzug ist festzustellen, dass aufseiten der Vergabekammern die Möglichkeiten die Durchführung von Vergabeverfahren durch externe Unternehmen durchführen zu lassen, positiv gewertet sind. Richtungsweisend dürfte dazu die Entscheidung der Vergabekammer Lüneburg, Beschluss vom 8. Mai 2018 - VgK-10/2018 heranzuziehen sein. Hier hat die Vergabekammer in Bezug auf eine Angebotsöffnung festgestellt, dass auch die Angebotsöffnung „outgesourct“" werden darf. Es bleibt zu hoffen, dass betroffene Auftraggeber vorbereitet in die Digitalisierung des Vergaberechts eintreten.

Heiner Osterhues

Rechtsanwalt in Köln und Redaktionsbeirat des Magazins Klinik Einkauf 